\title{
Erratum: Hygrometric Determination of Water Activities and Osmotic and Activity Coefficients of $\mathrm{NH}_{4}-\mathrm{KCl}-\mathrm{H}_{2} \mathrm{O}$ at $25^{\circ} \mathrm{C}$
}

The authors of this paper are Mohamed EL Guendouzi (Laboratoire de Chimie Physique, Département de Chimie, Faculté des Sciences Ben M'Sik, Université Hassan II Mohammedia, B.P 7955, Casablanca, Marco; email: elguendouzi@ yahoo.fr), Asmaa Benbiyi, Abderrahim Dinane, Rachid Azougen, and Abdelfetah Mounir rather than Abderrahim Dinane (Ecole Royale Navale, Département Universitaire, Boulevard Sour Jdid, Casablanca, Maroc; email: dinaneab@yahoo.fr) and Abdelfetah Mounir (Laboratoire de Chimie Physique, Département de Chimie, Faculté des sciences Ben M'sik, Université Hassan II Mohammedia, B.P. 7955, Casablanca, Maroc), as was listed.

The results on p. 399 of the activity coefficient were miscalculated and the values of the Pitzer mixing ionic parameters are not reliable, because the standard deviation $\sigma=0.0104$ is very large. The reported values of the parameters $\theta_{\mathrm{NH}_{4}^{+} \mathrm{K}^{+}}$and $\psi_{\mathrm{NH}_{4}^{+} \mathrm{K}^{+} \mathrm{Cl}^{-}}$, yield incorrect values of $\gamma_{\mathrm{NH}_{4} \mathrm{Cl}}$ and $\gamma_{\mathrm{KCl}}$. We have refined this calculation. The corrected values are: $\theta_{\mathrm{NH}_{4}^{+} \mathrm{K}^{+}}=-0.0272$ and $\psi_{\mathrm{NH}_{4}^{+} \mathrm{K}^{+} \mathrm{Cl}^{-}}=0.004866$ with $\sigma=0.0021$.

The corrected results are presented in Table I and Figures 1 and 2.

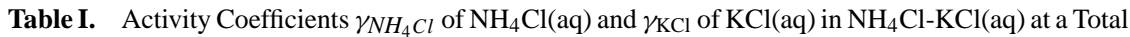
Ionic-Strength $I$ for Different Ionic Strength Fractions $y$ of $\mathrm{NH}_{4} \mathrm{Cl}$

\begin{tabular}{|c|c|c|c|c|c|c|c|c|c|c|c|c|c|c|}
\hline \multicolumn{3}{|c|}{$y=0$} & \multicolumn{3}{|c|}{$y=0.33$} & \multicolumn{3}{|c|}{$y=0.5$} & \multicolumn{3}{|c|}{$y=0.67$} & \multicolumn{3}{|c|}{$y=1$} \\
\hline$I$ & $\gamma / \mathrm{NH}_{4} \mathrm{Cl}$ & $\gamma_{\mathrm{KCl}}$ & $I$ & $\gamma \mathrm{NH}_{4} \mathrm{Cl}$ & $\gamma_{\mathrm{KCl}}$ & $I$ & $\gamma \mathrm{NH}_{4} \mathrm{Cl}$ & $\gamma_{\mathrm{KCl}}$ & $I$ & $\gamma / \mathrm{NH}_{4} \mathrm{Cl}$ & $\gamma_{\mathrm{KCl}}$ & $I$ & $\gamma / \mathrm{NH}_{4} \mathrm{Cl}$ & $\gamma_{\mathrm{KCl}}$ \\
\hline & 0695 & 685 & 0. & 0.692 & 0.689 & 4 & 0.670 & 0.671 & 0.3 & 8 & 0.692 & 0.3 & 0.685 & 0.696 \\
\hline 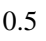 & 0.664 & 0.648 & 0.6 & 0.647 & 0.641 & 1.0 & 0.615 & 0.616 & 0.6 & 0.641 & 0.648 & 1.0 & 0.603 & 0.632 \\
\hline 1.0 & 0.632 & 0.602 & 0.9 & 0.626 & 0.617 & 2.0 & 0.589 & 0.594 & 0.9 & 0.617 & 0.626 & 2.0 & 0.571 & 0.622 \\
\hline 2.0 & 0.624 & 0.572 & 1.5 & 0.608 & 0.593 & 3.0 & 0.582 & 0.594 & 1.5 & 0.591 & 0.607 & 3.0 & 0.563 & 0.633 \\
\hline 3.0 & 0.639 & 0.569 & 3.0 & 0.607 & 0.584 & 4.0 & 0.580 & 0.603 & 3.0 & 0.575 & 0.606 & 4.0 & 0.562 & 0.649 \\
\hline 4.0 & 0.620 & 0.580 & 4.5 & 0.624 & 0.599 & 5.0 & 0.576 & 0.615 & 4.5 & 0.570 & 0.622 & 5.0 & 0.565 & 0.666 \\
\hline 4.5 & 0.675 & 0.589 & 6.0 & 0.640 & 0.621 & 6.0 & 0.568 & 0.628 & 5.7 & 0.565 & 0.636 & 6.0 & 0.567 & 0.680 \\
\hline
\end{tabular}




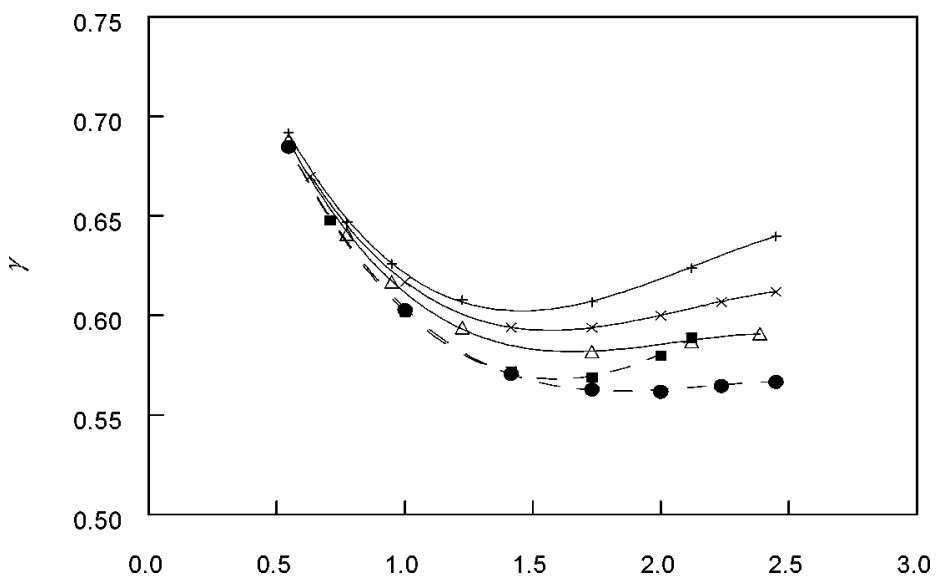

Fig. 1. Activity coefficients $\gamma_{\mathrm{NH}_{4} \mathrm{Cl}}$ of $\mathrm{NH}_{4} \mathrm{Cl}(\mathrm{aq})$ in $\mathrm{NH}_{4} \mathrm{Cl}-\mathrm{KCl}$ (aq) against the square root of total ionic strength $I^{1 / 2}$ at different ionic strength fractions $y$ of $\mathrm{NH}_{4} \mathrm{Cl}(\mathrm{aq}), \boldsymbol{\square}, y=0(\mathrm{KCl}) ;+, y=0.33 ; \times, y=0.50 ; \Delta, y=0.67 ; \bullet, y=1.0$ $\left(\mathrm{NH}_{4} \mathrm{Cl}\right)$.

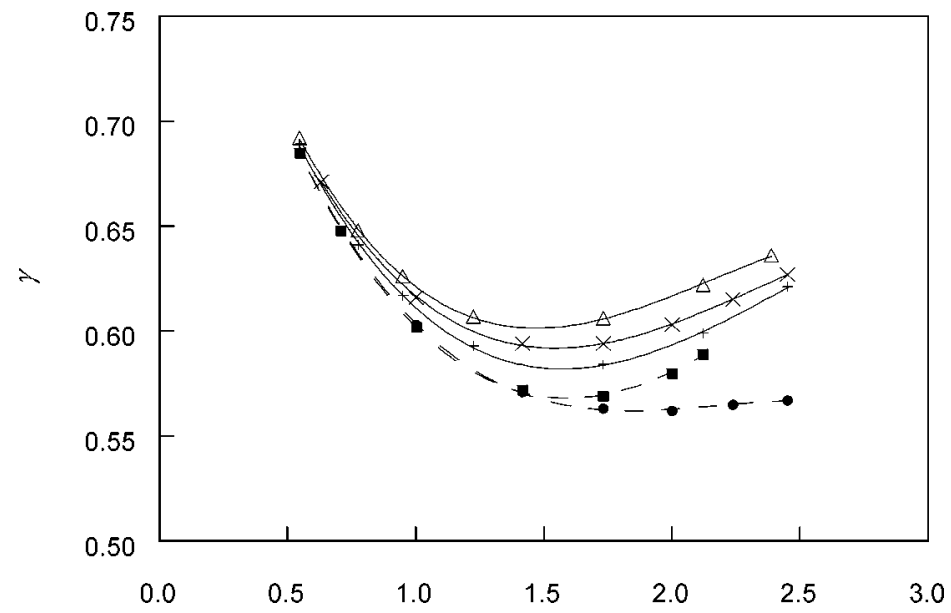

Fig. 2. Activity coefficients $\gamma_{\mathrm{KCl}}$ of $\mathrm{KCl}(\mathrm{aq})$ in $\mathrm{NH}_{4} \mathrm{Cl}-\mathrm{KCl}$ (aq) against the square root of total ionic strength $I^{1 / 2}$ at different ionic strength fractions $y$ of $\mathrm{NH}_{4} \mathrm{Cl}(\mathrm{aq})$. 口, $y=0(\mathrm{KCl}) ;+, y=0.33 ; \times, y=0.50 ; \Delta, y=0.67 ; \bullet, y=1.0\left(\mathrm{NH}_{4} \mathrm{Cl}\right)$. 